\title{
Comparaison des peuplements de diatomées épilithiques de ruisseaux acides en milieu forestier soumis à un amendement de dolomie (Massif Ardennais, Belgique)
}

\author{
A. Loncin 1,2 \\ L. Leclercq ${ }^{3}$ \\ M. $\mathrm{Carnol}^{4}$ \\ J. Remacle 4 \\ P. Vander Borght ${ }^{1}$
}

Mots clés : Bacillariophyceae, diatomées, amendement, ruisseaux, eaux acides, bioindicateurs, Eunotia, Ardenne.

Trois à cinq ans après un amendement de dolomie (carbonate calco-magnésien) réalisé en Belgique sur certains bassins versants forestiers du massif ardennais, les diatomées épilithiques ont été prélevées dans deux ruisseaux situés en zone amendée et dans deux ruisseaux en zone non amendée afin de déceler les éventuels changements induits dans les peuplements algaux. On observe des assemblages à Eunotia typiques des eaux acides. L'espèce dominante est Eunotia exigua, souvent accompagnée par $E$. rhomboidea ou E. bilunaris. Dans le cadre de cette étude, aucune influence propre à l'amendement n'a pu être mise en évidence par l'étude de ces bioindicateurs.

Comparison of epilithic diatom populations from acid streams in a forest catchment treated with dolomite (Ardenne, Belgium)

Keywords : Bacillariophyceae, diatoms, amendment, streams, acid waters, bioindicators, Eunotia, Ardenne.

Three to five years following application of dolomite (calcium magnesium carbonate) on some forest catchments in the belgian area of the Ardenne, epilithic diatoms were sampled in two streams running on treated sites and in two streams running on untreated sites in order to evaluate the possible changes in the algal population. Typical acid water assemblages with Eunotia were observed. The dominant species was Eunotia exigua often found with E. rhomboidea or E. bilunaris. In the present study, no influence of the treatment can be demonstrated using these bioindicators.

\section{Introduction}

En vue d'enrayer le phénomène de dépérissement forestier constaté sur le haut plateau ardennais en Belgique, des épandages de dolomie (3 t/ha de

\footnotetext{
1. Fondation Universitaire Luxembourgeoise, Laboratoire de Gestion des Eaux, avenue de Longwy 185, 6700 Arlon, Belgique. 2. Auteur correspondant : Centre de Recherche Public - Centre Universitaire, CREBS, avenue de la Faïencerie 162a, 1511 Luxembourg, Grand-Duché de Luxembourg.

3. Université de Liège, Station Scientifique des Hautes-Fagnes, rue de Botrange 137, 4950 Robertville, Belgique.

4. Université de Liège, Laboratoire d'Ecologie Microbienne, Institut de Botanique, B22, Sart Tilman, 4000 Liège, Belgique.
}

$\left.\mathrm{CaMg}\left(\mathrm{CO}_{3}\right)_{2}\right)$ et de sulfate de potassium $(200 \mathrm{~kg} / \mathrm{ha} \mathrm{de}$ $\mathrm{K}_{2} \mathrm{SO}_{4}$ ) ont été réalisés en forêt sur deux bassins versants de ruisseaux acides : d'une part au Waroneu (Hautes-Fagnes) en mai 1992 et d'autre part au Burhé (plateau de la Croix Scaille) en mai 1993 (Anonyme 1994). Deux autres bassins versants proches et de caractéristiques similaires ont été choisis pour observer l'évolution naturelle des écosystèmes, à la Robinette (Hautes-Fagnes) et à la Doucette (Plateau de la Croix Scaille). Des prélèvements de diatomées épilithiques, organismes considérés comme de bons indicateurs de la qualité des eaux acides (Symoens 1957, Battarbee 1984, Fabri \& Leclercq 1984, Stevenson et al. 1991, Lancaster et al. 1996), ont été effectués entre octobre 1996 et août 1997 dans dix stations de ces quatre ruisseaux. Ces organismes devraient nous permettre de dé- 
celer les différences ou similitudes entre les ruisseaux des deux régions ardennaises et les variations éventuellement induites dans l'écosystème rhithral par l'amendement, après une période de trois à cinq ans.

\section{Caractéristiques et localisation des sites étudiés}

Il a été montré (Fabri \& Leclercq 1984) que les ruisseaux de ces régions sont oligotrophes, très acides, riches en acides humiques et peu minéralisés, souvent bien pourvus en sulfates. Les quatre bassins versants choisis sont similaires du point de vue géologique : roche cambrienne composée de quartzites et de quartzo-phyllades (Anonyme 1994). La vallée du ruisseau Waroneu est plantée en partie d'essences feuillues alors que les trois autres bassin versants sont entièrement enrésinés de Picea abies (L.) Karst.

Les deux ruisseaux fagnards, Waroneu et Robinette, présentent une confluence de deux branches (Fig.1). $\mathrm{Au}$ Waroneu, trois points de prélèvement ont été sélectionnés : branche Nord (station Wn), branche Sud (sta-

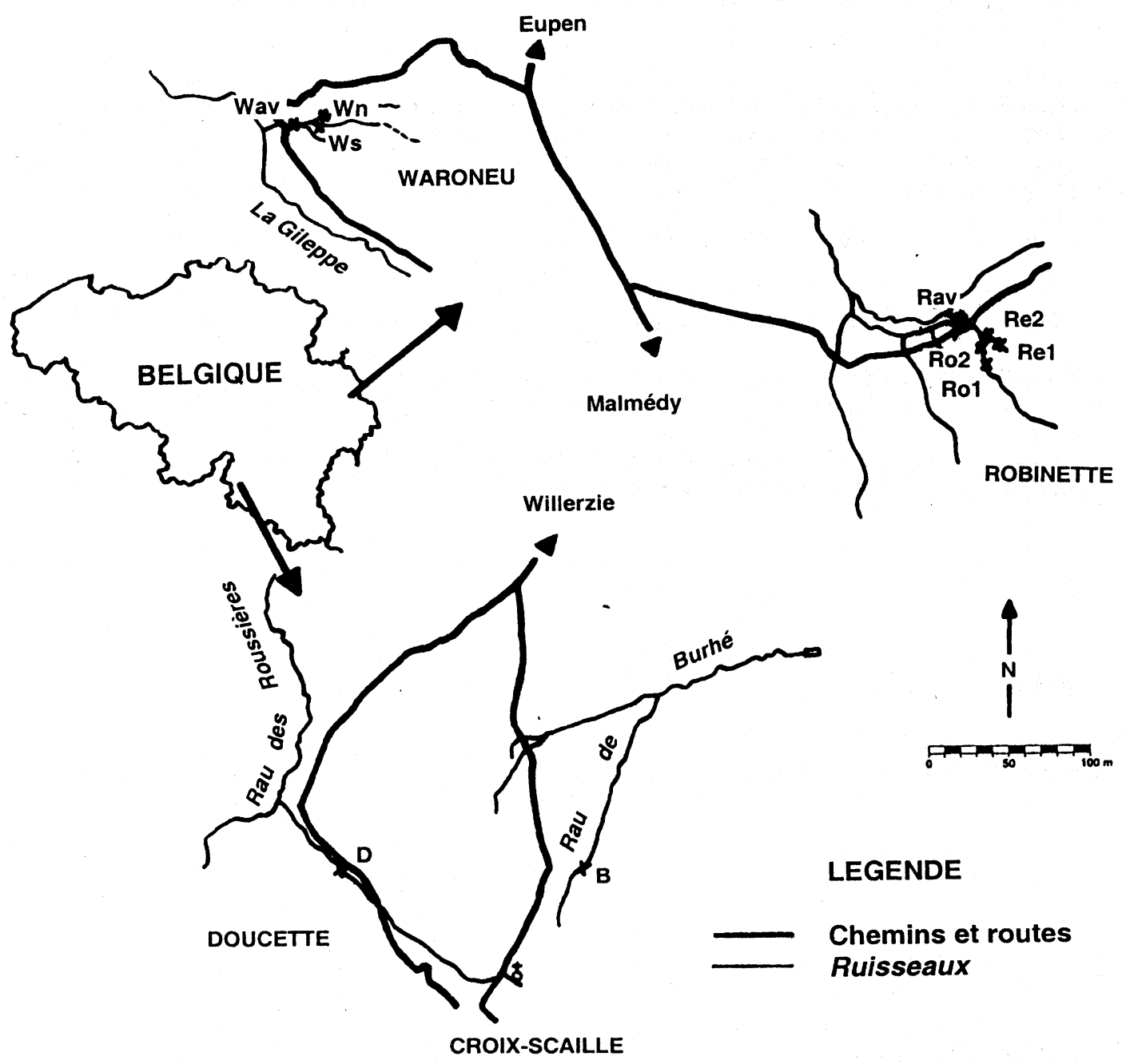

Fig. 1. Localisation des sites étudiés.

Fig. 1. Location of the studied sites. 
tion Ws) et aval de la confluence (station Wav). A la Robinette, cinq stations ont été choisies : deux sur la branche Ouest (stations Ro1 et Ro2), deux sur la branche Est (stations $\operatorname{Re} 1$ et $\operatorname{Re} 2$ ) et une à l'aval de la confluence (station Rav). Re1 et Ro1 représentent les sites de prélèvement le plus en amont, localisés dans une zone de coupes à blanc et sont donc bien éclairés, tandis que les stations $\operatorname{Re} 2$ et Ro2 sont situées en milieu forestier couvert. Une seule station de prélèvement a été échantillonnée pour chacun des ruisseaux du plateau de la Croix Scaille, le Burhé (station B) et la Doucette (station D).

\section{Matériel et méthodes}

Les dix stations de prélèvement ont été échantillonnées à huit reprises : en octobre et décembre 1996 et chaque mois de mars à août 1997. Le prélèvement consiste à brosser vigoureusement la face supérieure de quelques pierres ou cailloux choisis dans la zone lotique. L'échantillon, correspondant à une surface totale d'environ $500 \mathrm{~cm}^{2}$, est récupéré par rinçage des pierres et fixé au formol. La matière organique est ensuite éliminée par traitement à l'acide nitrique concentré à chaud. Après plusieurs lavages de l'acide à l'eau distillée, la suspension ne contient plus que les frustules siliceux des diatomées. Après séchage, quelques gouttes de cette suspension sont montées entre lame et lamelle avec du Naphrax. Toutes les espèces présentes sont alors déterminées au microscope optique. $\mathrm{Au}$ moins 500 individus sont comptés par préparation. Les espèces présentes mais n'apparaissant pas dans le comptage se voient attribuer par convention une abondance relative de $0,1 \%$. Les listes floristiques ont été traitées par classification hiérarchique (dendrogramme de similarité) grâce au logiciel Primer v.4.0 (1996) du Plymouth Marine Laboratory.

\section{Résultats}

Pour l'ensemble des quatre ruisseaux, un total de 106 taxons a été recensé (Tableau1). De nombreux taxons ont été répertoriés dans l'ensemble des dix stations étudiées. Quinze taxons sont considérés comme principaux car ils présentent une abondance relative supérieure à $1 \%$ dans au moins un échantillon. Le tableau 2 reprend l'abondance relative par échantillon de ces 15 taxons. Parmi ceux-ci, le genre Eunotia représente généralement plus de $80 \%$ du peuplement, avec principalement $E$. bilunaris, E. exigua, E. rhomboidea et $E$. tenella. La dominance d'Eunotia exigua est nette dans toutes les stations étudiées et durant toute l'année. Cette espèce est généralement accompagnée par
E. rhomboidea, sauf dans la branche Ouest de la Robinette où elle est plus souvent associée à $E$. bilunaris. Ce denier taxon est également bien présent au Burhé, à l'aval de la Robinette et en Re1. Il arrive exceptionnellement en octobre 1996 et en août 1997 que Tabellaria flocculosa soit abondante dans le Burhé.

La plus grande diversité est observée en Re2, tandis que la station $\mathrm{D}$ est la moins diversifiée.

Le dendrogramme des indices de similarité de BrayCurtis calculés à partir du tableau 2 (Fig.2) montre un clivage des échantillons en trois groupes à $70 \%$ seulement. Les différents relevés sont donc très semblables, ce qui paraît logique vu la dominance d'un seul ou plus rarement de deux ou trois taxons : Eunotia exigua, E. rhomboidea et $E$. bilunaris. Le groupe 1 reprend principalement les échantillons du Waroneu, caractérisés par la présence marquée d'Achnanthes helvetica et la faible représentation des deux variétés d'Eunotia paludosa. Le groupe 2 réunit la branche Ouest de la Robinette à quelques échantillons de la Robinette aval et du Burhé. Le groupe 3 est composé de la branche Est de la Robinette, de la Doucette et des autres échantillons du Burhé. Les échantillons des groupes 2 et 3 sont caractérisés par une très faible représentation d'Achnanthes helvetica et la présence nette d'Eunotia paludosa, surtout dans sa variété nominale. Aucune différence n'apparaît entre les sites bien éclairés de la Robinette (Re1 et Ro1) et les sites couverts ( $\operatorname{Re} 2$ et Ro2). Le Waroneu semble s'individualiser par rapport à la Robinette. Cette observation trouve peut-être son origine dans la couverture forestière des bassins versants. En effet, tous sont plantés d'épicéas (Picea abies (L.) Karst.) sauf celui du Waroneu qui est couvert de feuillus (Fagus sylvatica (L.)) en aval. Malgré ces considérations, il ne faut pas perdre de vue que tous les échantillons sont similaires à plus de $70 \%$.

\section{Discussion et conclusion}

Les taxons principaux observés (Tableau 2) sont caractéristiques des milieux acides (Alles et al. 1991, Coring 1993, Kwandrans 1993, Pierre 1996). L'espèce majoritaire, Eunotia exigua, est connue comme acidobionte et très résistante aux métaux lourds, notamment l'aluminium (Van Dam 1981, Alles et al. 1991). Or, les ruisseaux étudiés ont un $\mathrm{pH}$ proche de 4,0 et des teneurs en aluminium assez élevées, de 0,6 à $1,9 \mathrm{mg}$ $\mathrm{Al}^{+++} / 1$ (Carnol 1997). Cet Eunotia trouve donc son optimum de développement et domine dans ces ruisseaux ardennais acides alors que les autres taxons comme E. paludosa et E. bilunaris supportent probablement moins bien la présence d'aluminium dans l'eau (Alles et al. 1991). 
Tableau 1. Composition de la flore diatomique et nombre total de taxons recensés dans les dix stations de prélèvements.

Table 1. Composition of the diatom flora and total number of taxa found at the ten sites.

\begin{tabular}{|c|c|c|c|c|c|c|c|c|c|c|}
\hline & Rav & Re1 & Re2 & Ro1 & $\mathbf{R o 2}$ & Wav & $W_{n}$ & Ws & $\mathbf{B}$ & D \\
\hline Achnanthes helvetica (Hustedt) Lange-Bertalot & + & + & + & & + & + & + & + & + & + \\
\hline Achnanthes lanceolata (Brébisson) Grunow & + & + & & & + & + & + & + & + & \\
\hline Achnanthes marginulata Grunow & & & & & & + & & + & & \\
\hline Achnanthes minutissima Kützing & + & + & + & + & + & & & + & + & + \\
\hline Achnanthes oblongella Oestrup & & & $+\cdots$ & & & & & & & \\
\hline Amphora inariensis Krammer & & & & & & & & & + & \\
\hline Brachysira brebissonii Ross & & + & + & & & & & & + & + \\
\hline Brachysira serians (Brèbisson) Round et Mann & & & & & & + & & & + & \\
\hline Caloneis bacillum (Grunow) Cleve & + & + & + & + & + & + & + & + & + & + \\
\hline Cocconeis placentula var. euglypta (Erhrenberg) Grunow & & & + & & & & + & + & + & \\
\hline Cyciotella meneghiniana Kützing & & & & & & & & & & + \\
\hline Cymbella aequalis Smith : & & & + & & & & & & & + \\
\hline Cymbella falaisensis (Grunow) Krammer \& Lange-Bertalot & & & + & + & & & & & + & \\
\hline Cymbella hybrida var. lanceolata Krammer & & & & & & & + & & & \\
\hline Cymbella naviculiformis Auerswald & & + & + & & & + & & & & \\
\hline Diadesmis contenta (Grunow ex Van Heurck) Mann & & & + & & & & + & & $:$ & \\
\hline Diatoma moniliformis Kützing & & & & & & & & & + & \\
\hline Encyonema minutum (Hilse in Rabenhorst) Mann & & & & + & + & + & & + & & \\
\hline Encyonema perpusillum (Cleve) Mann & & & & & & + & & & + & + \\
\hline Eunotia bilunaris (Erhrenberg) Mills & + & + & + & + & + & + & + & + & + & + \\
\hline Eunotia bilunaris var. mucophila Lange-Bertalot, Nörpel \& Alles & & + & + & & + & & & + & & + \\
\hline Eunotia exigua (Brèbisson) Rabenhorst & + & + & + & + & + & + & + & + & + & + \\
\hline Eunotia fallax Cleve & & + & + & + & & & & & & \\
\hline Eunotia incisa Gregory & + & + & + & & + & + & + & + & + & + \\
\hline Eunotia minor (Kützing) Grunow & + & + & + & & & + & & + & + & \\
\hline Eunotia paludosa Grunow & + & + & + & + & + & + & + & + & + & + \\
\hline Eunotia paludosa var. trinacria (Krasske) Nörpel \& Alles & + & + & + & + & + & + & + & & + & + \\
\hline Eunotia praerupta Ehrenberg & & & + & & & & + & & & \\
\hline Eunotia Tomboidea Hustedt & + & + & + & + & + & + & + & + & + & + \\
\hline Eunotia silvahercynia Nörpel, Van Sull \& Lange-Bertalot & & & & & & & & & + & \\
\hline Eunotia sudetica Muller & + & + & + & + & + & + & + & + & + & \\
\hline Eunotia tenella (Grunow) Hustedt & + & + & + & + & + & + & + & + & + & + \\
\hline Fallacia insociabilis (Krasske) Mann & & & + & & + & & & & & \\
\hline Fragilaria capucina var. vaucheriae (Kützing) Lange-Bertalot & & & & & & & & + & & + \\
\hline Fragilariforma virescens (Ralfs) Williams \& Round & & & & + & & + & + & & + & + \\
\hline Frustulia Momboides var. saxonica (Rabenhorst) De Toni & + & + & + & + & + & + & + & + & + & + \\
\hline Frustulia vulgaris (Thwaites) De Toni & & & & + & & + & & & & \\
\hline Gomphonema micropus Kützing & + & & & & + & + & & & & \\
\hline Gomphonema olivaceum (Horneman) Brébisson & & & & & & + & + & & + & \\
\hline Gomphonema parvulum var. exilissimum Grunow & + & + & + & + & & + & + & + & & \\
\hline Hantzschia amphyoxis (Erhenberg) Grunow & + & + & + & & . & & + & + & + & \\
\hline Luticola mutica (Kützing) Mann & + & & & & + & & & + & + & \\
\hline Luticola muticopsis (Van Heurck) Mann & & & & & + & & & & & \\
\hline Luticola nivalis (Ehrenberg) Mann & & & + & & & & & & & \\
\hline Meridion circulare (Greville) Agardh & & & & & & & & + & & \\
\hline
\end{tabular}


Tableau 1. Suite

Table 1. Continued

Navicula atomus (Kützing) Grunow

Navicula bryophila Boye Petersen

Navicula cincta (Ehrenberg) Ralfs

Navicula cryptocephala Kützing

Navicula fossalis Krasske

Navicula fossalis var. obsidialis (Hustedt) Lange-Bertalot

Navicula gallica var. perpusilla (Grunow) Lange-Bertalot

Navicula gregaria Donkin

Navicula krasskei Hustedt

Navicula lanceolata (Agardh) Ehrenberg

Navicula lapidosa Krasske

Navicula mediocris Krasske

Navicula minima Grunow

Navicula parsura Hustedt

Navicula recens (Lange-Bertalot) Lange-Bertalot

Navicula soehrensis Krasske

Navicula subminuscula Manguin

Navicula tenelloides Hustedt

Neidium alpinum Hustedt

Neidium ampliatum (Ehrenberg) Krammer

Neidium carterii Krammer

Neidium hercynicum Mayer

Nitzschia acidoclinata Lange-Bertalot

Nitzschia amphibia Grunow

Nitzschia capitellata Hustedt

Nitzschia dissipata (Kützing) Grunow

Nitzschia palea var. debilis (Kützing) Grunow

Nitzschia solgensis Cleve-Euler

Pinnularia appendiculata (Agardh) Cleve

Pinnularia biceps f. minutissima (Hustedt) Cleve

Pinnularia borealis var. scalaris (Ehrenberg) Rabenhorst

Pinnularia carminata Barber \& Carter

Pinnularia mesolepta (Ehrenberg) Smith

Pinnularia microstauron (Ehrenberg) Cleve

Pinnularia obscura Krasske

Pinnularia rupestris Hantzsch

Pinnularia schoenfelderi Krammer

Pinnularia silvatica Petersen

Pinnularia subcapitata Gregory

Pinnularia subcapitata var. elongata Krammer

Pinnularia subcapitata var. subrostrata Krammer

Pinnularia subgibba Krammer

Pinnularia submicrostauron Schroeter

Pinnularia sudetica (Hilse) Hilse

Pinnularia vinidis (Nitzsch) Ehrenberg
Rav Re1 Re2 Ro1 Ro2 Wav Wn Ws B D

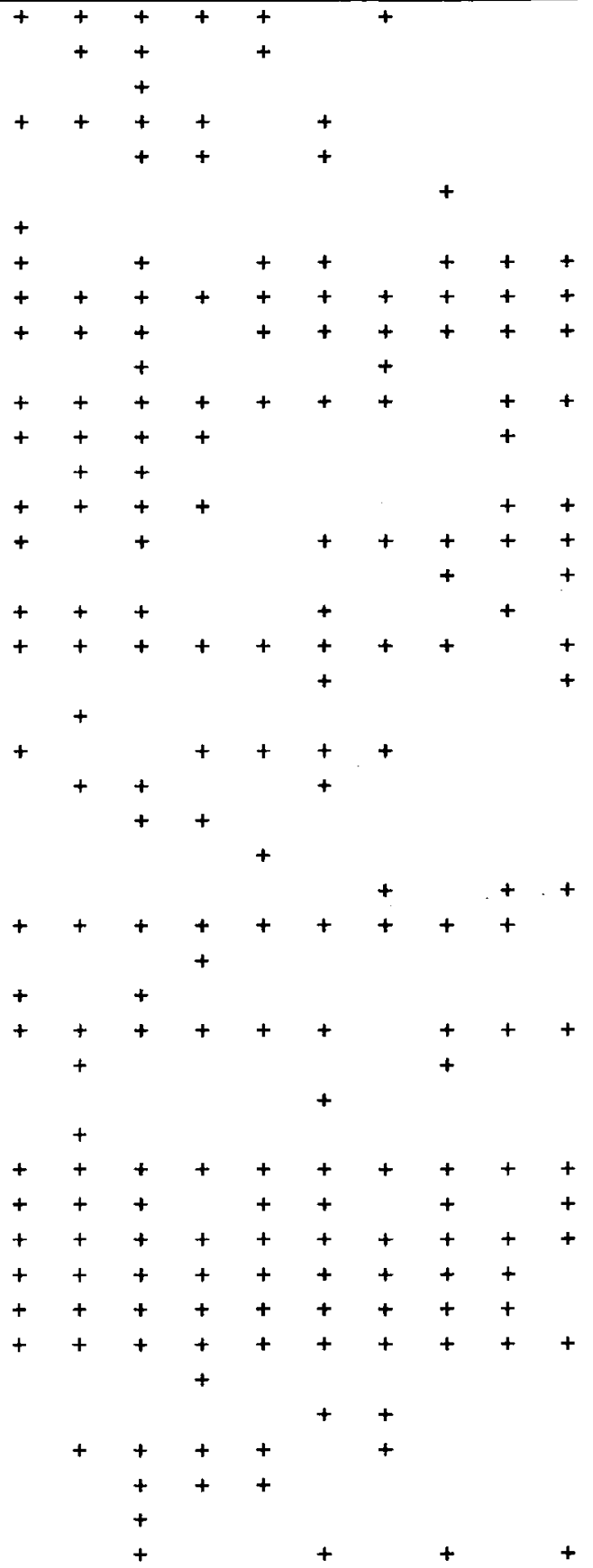


Tableau 1. Suite

Table 1. Continued

\begin{tabular}{|c|c|c|c|c|c|c|c|c|c|c|}
\hline & Rav & $\operatorname{Re} 1$ & $\operatorname{Re} 2$ & Ro1 & Ro2 & Wav & Wn & Ws & B & D \\
\hline Placoneis elginensis (Gregory) Cox & & + & + & & & & & + & & \\
\hline Reimeria sinuata (Gregory) Kociolek \& Stoermer & + & + & & & & & & & & \\
\hline Rhoicosphenia abbreviata (Agardh) Lange-Bertalot & & + & & & & & & & + & \\
\hline Stauroneis anceps Ehrenberg & & & + & & & + & & & & \\
\hline Stauroneis phoenicenteron (Nitzsch) Ehrenberg & & & & & & + & & & & \\
\hline Stauroneis thermicola (Petersen) Lund & + & + & + & + & & + & + & & & \\
\hline Stenopterobia delicatissima (Lewis) Brébisson & & & & & & + & & & & \\
\hline Surirella angusta Kützing & & + & & & & & & & & \\
\hline Surirella brebissonii var. kuetzingii Krammer \& Lange-Bertalot & + & & & & & & & + & & \\
\hline Surirella linearis Smith & & + & + & & & & + & & & \\
\hline Surirella minuta Brébisson & & & & & & & & + & & \\
\hline Surirella roba Leclercq & & + & + & & + & + & + & + & & \\
\hline Surirella tenuis Mayer & + & & & & & & + & & + & \\
\hline Tabellaria flocculosa (Roth) Kützing & + & + & + & + & + & + & + & + & + & + \\
\hline Tabellaria ventricosa Kützing & & + & + & & + & + & + & + & + & + \\
\hline Nombre de taxons présents & 45 & 53 & 65 & 39 & 41 & 53 & 43 & 45 & 46 & 36 \\
\hline
\end{tabular}

La grande diversité taxonomique observée en Re2 trouve peut-être son explication dans les perturbations subies par cette branche du ruisseau, suite aux coupes forestières en amont du site. Le lit du ruisseau a été partiellement obstrué par des troncs, des branchages et de grandes quantités d'aiguilles d'épicéas. Il s'en est suivi un ralentissement de l'écoulement et un développement important de bryophytes. Cette branche du ruisseau a donc évolué vers un système de plus en plus lentique. La matière organique accumulée ayant entamé un processus de dégradation, il est possible que la trophie de l'eau ait augmenté, favorisant la richesse spécifique. Cette diversité a pu être favorisée par la complexation de l'aluminium par les acides humiques amenés dans le ruisseau par ruissellement des terrains environnants (Alles et al. 1991).

Il apparaît donc que tous les cours d'eau présentent des peuplements diatomiques à Eunotia très semblables du fait de leur très forte acidité et de leur oligotrophie. De légères différences se manifestent dans la diversité taxonomique, sans doute dues à divers facteurs écologiques difficiles à appréhender (vitesse du courant, luminosité, broutage,...), ou simplement liées à la variabilité d'échantillonnage.

En raison des caractéristiques géologiques de leur bassin versant déterminant des eaux très acides et oli- gotrophes, les quatre ruisseaux observés dans ces deux régions ardennaises présentent des peuplements de diatomées similaires, dominés par le genre Eunotia et principalement par Eunotia exigua.

En ce qui concerne les amendements calco-magnésiens effectués au Waroneu en 1992 et au Burhé en 1993, nous n'en constatons aucune trace dans les peuplements diatomiques en 1996 et 1997 . On peut raisonnablement penser que, les quantités épandues étant modestes, l'influence éventuelle de ce chaulage sur l'écosystème rhithral est devenu imperceptible après trois à cinq ans.

\section{Remerciements}

Cette étude a pu être réalisée grâce au mandat de Collaborateur scientifique du F.N.R.S. alloué à l'auteur dans le cadre de la convention F.R.F.C. 2.457195 «Evaluation de la résilience d'écosystèmes forestiers à des perturbations d'origine anthropique : impact sur la phytocénose et la microbiocénose» et aux projets pilote Région Wallonne - Union européenne «Restauration des forêts endommagées et suivi des effets» et «Étude comparée de paramètres écosystémiques et de l'évolution phytosanitaire de peuplements situés dans des couples de bassins versants». Nous tenons à remercier M. Luc Ector pour ses judicieux conseils lors de la lecture du manuscrit, et M. Henry-Michel Cauchie pour son aide précieuse lors de l'utilisation du programme statistique. 


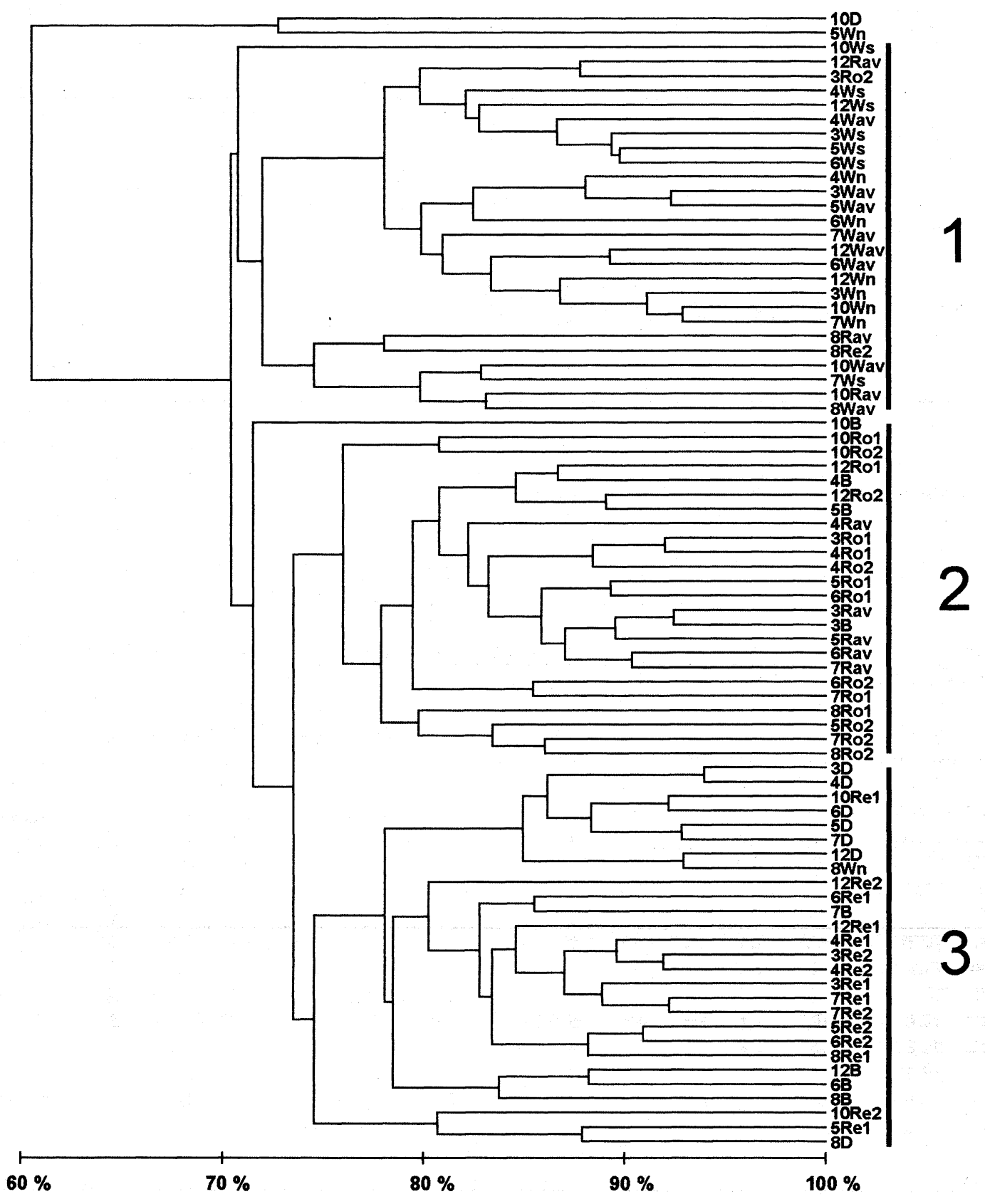

Fig. 2. Dendrogramme des échantillons établi sur base du tableau 2 (indices de similarité de Bray-Curtis). Le nombre précédant la station représente le mois du prélèvement.

Fig. 2. Clustering of samples based on table 2 (Bray-Curtis similarity). The number before the station represents the month of sampling. 
Tableau 2. Abondance relative des taxons principaux ( $\geq 1 \%$ dans au moins un échantillon) exprimée en $\%$. Le nombre précédant la station correspond au mois du prélèvement.

Table 2. Relative abundance of the main taxa ( $\geq 1 \%$ in at least one sample) expressed as $\%$. The number before the station represents the month of sampling.

\begin{tabular}{|c|c|c|c|c|c|c|c|c|c|c|c|c|c|c|c|}
\hline & 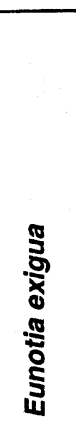 & 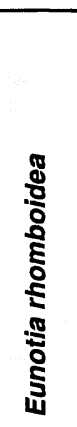 & 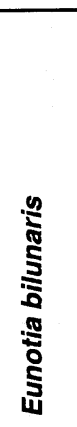 & 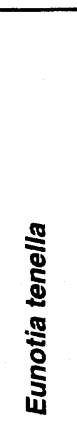 & 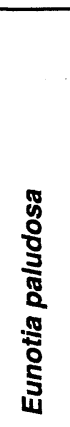 & 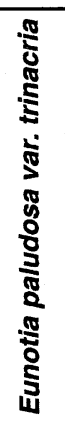 & 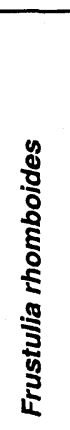 & 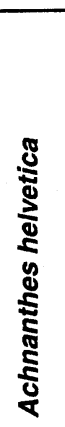 & 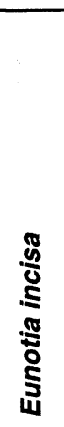 & 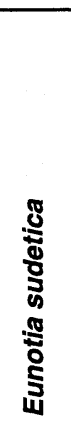 & 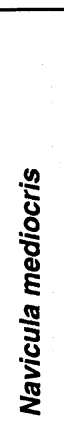 & 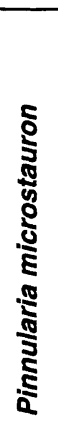 & 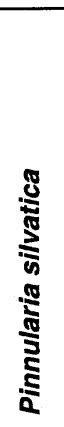 & 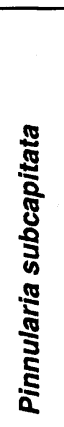 & 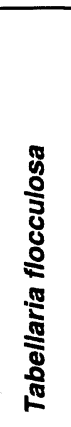 \\
\hline 10Rav & 70,3 & 11,8 & 8,2 & 0,1 & 0,1 & $\cdot$ & 6,7 & 0,1 & - & 0,6 & - & - & 0,1 & 1,4 & - \\
\hline 10Re1 & 87,8 & 2,6 & 2,2 & 0,2 & 2,6 & - & 0,1 & 0,1 & 1,2 & - & 0,1 & 0,1 & - & 0,2 & 0,9 \\
\hline 10Re2 & 75,2 & 8,1 & 2,3 & - & 0,1 & - & 0,9 & 0,1 & 0,1 & 10,5 & 0,1 & 0,2 & 0,2 & 0,2 & 0,1 \\
\hline 10Ro1 & 95,0 & 0,1 & 2,7 & - & - & 0,1 & 1,0 & - & - & - & 0,1 & - & 0,1 & 0,4 & - \\
\hline 10Ro2 & 76,6 & 2,6 & 6,9 & - & - & 0,1 & 10,1 & - & - & 0,1 & 0,1 & 0,1 & 1,6 & 1,2 & - \\
\hline $10 \mathrm{Wav}$ & 59,8 & 26,6 & 3,3 & 3,3 & 0,4 & - & 0,9 & 0,4 & - & - & - & - & $\cdot$ & 2,5 & - \\
\hline $10 \mathrm{Wn}$ & 85,0 & 8,6 & 0,5 & 0,4 & - & - & 0,1 & 1,0 & - & - & - & 0,1 & 0,1 & 2,9 & 0,1 \\
\hline $10 \mathrm{Ws}$ & 53,1 & 1,5 & - & 31,6 & 1,0 & - & 1,0 & 0,4 & - & 1,0 & - & 0,1 & 1,3 & 4,4 & 0,1 \\
\hline $10 \mathrm{~B}$ & 51,5 & 2,4 & 10,4 & 0,1 & - & - & 6,3 & 0,1 & - & - & 0,4 & 0,1 & 0,2 & 0,4 & 27,7 \\
\hline 10D & 95,9 & 0,2 & 0,4 & - & - & - & 0,1 & - & 0,1 & - & - & - & - & 0,2 & 3,0 \\
\hline 12Rav & 91,8 & 2,6 & 2,4 & 1,3 & 0,2 & - & 0,1 & - & 0,1 & 0,1 & - & 0,1 & 0,1 & 0,1 & 0,1 \\
\hline 12Re1 & 60,7 & 14,4 & 2,5 & 1,4 & 11,4 & 1,4 & 0,2 & - & 1,6 & 0,4 & 1,0 & 1,0 & 0,1 & 0,8 & - \\
\hline 12Re2 & 72,5 & 2,3 & 5,4 & 0,1 & 4,2 & 0,6 & - & - & 1,0 & 9,2 & 0,4 & 0,2 & - & 1,3 & 0,2 \\
\hline 12Ro1 & 90,5 & 0,6 & 1,8 & 3,6 & - & 0,2 & 0,2 & - & - & - & 0,1 & - & 0,8 & 1,2 & 0,1 \\
\hline 12Ro2 & 78,2 & 1,5 & 8,3 & 3,2 & - & 2,5 & 0,9 & - & 0,4 & - & 0,1 & 0,1 & 0,6 & 1,7 & 0,2 \\
\hline $12 \mathrm{Wav}$ & 89,3 & 7,8 & 0,5 & 0,4 & - & - & 0,2 & 0,1 & 0,1 & - & - & 0,2 & 0,1 & 0,1 & - \\
\hline $12 \mathrm{Wn}$ & 94,3 & 3,3 & 0,1 & 0,1 & - & - & 0,1 & 0,6 & - & 0,1 & 0,1 & 0,2 & 0,1 & 0,1 & 0,1 \\
\hline $12 \mathrm{Ws}$ & 76,7 & 1,3 & 0,6 & 17,6 & - & 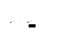 & 0,1 & 0,2 & 0,1 & 1,3 & - & 0,1 & 0,1 & 0,2 & 0,1 \\
\hline $12 B$ & 74,0 & 5,1 & 8,9 & 1,6 & 3,0 & 0,8 & 2,1 & - & 0,4 & - & - & 0,1 & 0,2 & 1,0 & 1,4 \\
\hline 12D & 78,6 & 3,3 & 4,0 & 0,6 & 1,9 & 0,1 & 0,1 & - & 2,5 & - & 0,2 & - & - & 0,4 & 7,4 \\
\hline 3Rav & 78,6 & 4,2 & 8,2 & 2,0 & 1,2 & - & 2,0 & - & $=$ & - & 0,4 & 0,1 & 1,2 & 1,4 & 0,1 \\
\hline 3Re1 & 47,3 & 31,0 & 9,0 & 1,3 & 4,0 & - & 0,4 & - & 0,2 & 0,1 & 0,2 & 0,7 & 0,1 & 2,4 & 1,5 \\
\hline 3Re2 & 75,1 & 9,2 & 1,6 & 1,4 & 3,0 & - & 0,2 & - & 4,6 & 0,1 & 1,0 & 0,2 & 0,1 & 1,4 & 0,2 \\
\hline 3Ro1 & 82,1 & 0,8 & 5,2 & 3,4 & 3,6 & - & 0,5 & - & - & - & - & - & 2 & 0,6 & 0,1 \\
\hline 3Ro2 & 93,2 & 0,2 & 3,2 & 1,2 & 0,1 & - & 0,4 & - & - & 0,1 & - & 0,1 & 0,6 & 0,2 & - \\
\hline 3 Wav & 96,6 & 0,2 & 0,1 & 0,8 & 0,1 & - & 0,1 & 0,4 & - & - & - & - & 0,1 & 0,4 & 0,1 \\
\hline $3 W n$ & 94,0 & 0,8 & 0,4 & 0,1 & - & - & 0,1 & 2,4 & - & - & - & 0,1 & 0,1 & 0,6 & 0,2 \\
\hline $3 W s$ & 89,2 & 3,4 & 0,4 & 3,0 & 0,6 & - & 0,2 & 1,0 & - & 0,4 & - & - & 0,2 & 0,6 & 0,1 \\
\hline 3B & 78,2 & 8,7 & 6,0 & 1,9 & 0,6 & 0,1 & 2,4 & - & - & - & 0,9 & 0,1 & 0,1 & 0,7 & 0,1 \\
\hline 3D & 96,7 & 0,6 & 1,0 & 0,1 & 0,8 & - & 0,1 & - & 0,1 & - & 0,1 & - & - & 0,1 & 0,1 \\
\hline
\end{tabular}


Tableau 2. Suite.

Table 2. Continued.

\begin{tabular}{|c|c|c|c|c|c|c|c|c|c|c|c|c|c|c|c|}
\hline & 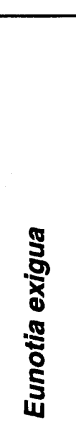 & 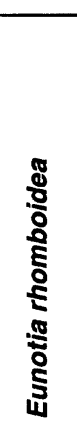 & 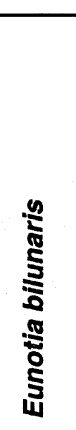 & 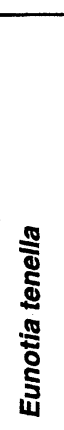 & 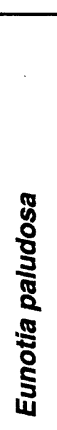 & 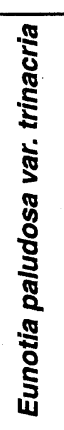 & 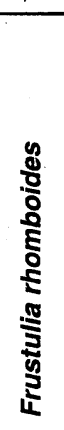 & 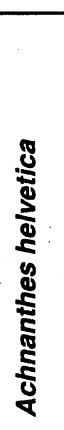 & 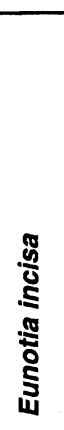 & 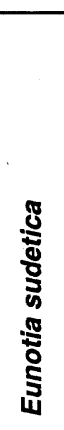 & 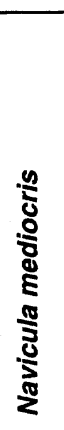 & 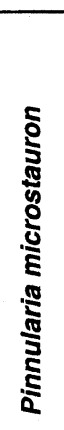 & 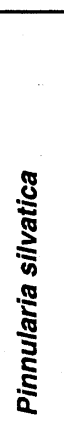 & 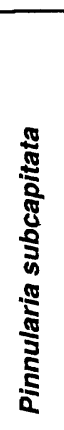 & 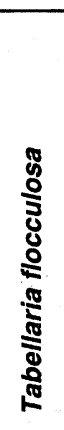 \\
\hline 4Rav & 56,6 & 28,5 & 3,8 & 0,6 & 1,8 & - & 6,6 & - & - & - & 0,1 & 0,2 & 1,2 & 0,2 & - \\
\hline 4Re1 & 76,8 & 2,4 & 3,4 & 2,6 & 8,0 & - & 0,1 & 0,2 & 1,2 & - & 2,2 & 0,1 & 0,2 & 0,8 & 0,4 \\
\hline 4Re2 & 80,3 & 2,4 & 5,2 & 1,2 & 3,4 & - & 0,1 & - & 2,2 & - & 0,6 & 0,4 & 0,2 & 1,2 & 0,2 \\
\hline 4Ro1 & 81,6 & 1,0 & 5,2 & 3,4 & 2,2 & - & 1,4 & - & - & - & 0,1 & - & 2 & 2,0 & - \\
\hline 4Ro2 & 79,0 & 2,4 & 7,2 & 0,6 & 6,2 & - & 2,0 & - & - & - & 0,4 & - & 0,6 & 0,4 & - \\
\hline 4Wav & 92,7 & 0,4 & 0,2 & 4,6 & 0,1 & - & 0,2 & 0,6 & - & 0,1 & 0,1 & - & 0,2 & 0,2 & - \\
\hline $4 W n$ & 97,4 & 0,2 & 0,1 & 0,6 & - & - & - & 0,6 & - & - & - & - & 0,1 & 0,4 & - \\
\hline $4 \mathrm{Ws}$ & 92,1 & 1,8 & 0,2 & 3,8 & 0,2 & - & 0,1 & 0,2 & 1,0 & 0,1 & - & - & - & 0,1 & - \\
\hline 4B & 72,4 & 4,5 & 6,7 & 3,3 & - & 0,2 & 1,2 & 0,1 & 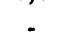 & - & 0,6 & - & 0,2 & 0,8 & 0,6 \\
\hline 4D & 96,0 & 0,4 & 0,8 & 0,8 & 0,2 & - & 0,1 & - & 0,4 & - & 0,1 & - & - & 0,1 & 0,2 \\
\hline 5Rav & 80,6 & 1,0 & 14,0 & 1,0 & 1,0 & - & 0,4 & - & - & - & 0,2 & 0,1 & 0,2 & 0,2 & 0,1 \\
\hline 5Re1 & 87,1 & 4,2 & 4,2 & - & 0,1 & - & 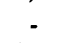 & 0,2 & 0,1 & - & 0,2 & 0,1 & - & 3,0 & 0,1 \\
\hline 5Re2 & 74,6 & 9,2 & 6,6 & 0,1 & 3,1 & - & 0,1 & - & - & 0,1 & 0,4 & 0,6 & - & 3,3 & 0,1 \\
\hline 5Ro1 & 88,7 & 0,4 & 6,6 & 0,7 & 0,2 & 0,6 & 0,8 & - & - & 0,1 & 0,2 & 0,1 & 0,6 & 0,1 & 0,1 \\
\hline 5Ro2 & 78,3 & - & 17,4 & 0,6 & 0,1 & 1,0 & 0,2 & 0,1 & - & - & - & 0,1 & 0,4 & 1,0 & 0,1 \\
\hline 5 Wav & 95,6 & 0,6 & 0,8 & 0,8 & 0,1 & - & 0,2 & 0,4 & - & - & - & - & 0,1 & 0,2 & - \\
\hline $5 W n$ & 98,3 & 0,1 & 0,1 & - & - & - & - & 0,6 & - & - & - & - & - & 0,2 & $\cdot$ \\
\hline $5 \mathrm{Ws}$ & 87,7 & 1,4 & 0,6 & 7,3 & - & - & 0,2 & 0,1 & - & 0,1 & - & - & 0,2 & 0,4 & 0,1 \\
\hline $5 B$ & 68,5 & 14,4 & 9,2 & 2,8 & - & 0,2 & 1,8 & 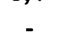 & 0,2 & 0,1 & 0,1 & 0,1 & 0,2 & 0,6 & 0,4 \\
\hline 5D & 94,8 & 0,6 & 1,0 & 1,2 & 0,6 & - & 0,1 & - & - & - & 0,1 & 0,1 & - & 0,2 & 1,0 \\
\hline 6Rav & 78,0 & 2,2 & 9,8 & 2,4 & 0,4 & 1,2 & 0,9 & - & - & - & 0,2 & 0,4 & 1,6 & 1,8 & - \\
\hline 6Re1 & 85,0 & 3,6 & 5,2 & 0,4 & 1,4 & - & - & - & - & - & 0,1 & 0,8 & 0,1 & 2,0 & 0,4 \\
\hline 6Re2 & 78,7 & 6,2 & 8,6 & 0,2 & 2,4 & - & 0,2 & 0,1 & - & 0,2 & 0,2 & 0,4 & - & 1,4 & - \\
\hline 6Ro1 & 84,7 & 0,1 & 8,2 & 1,4 & - & 0,4 & 0,9 & - & - & - & 0,2 & 0,1 & 2,2 & 1,0 & 0,1 \\
\hline 6Ro2 & 81,8 & 0,1 & 10,2 & 1,8 & 0,6 & 3,0 & 0,5 & - & - & - & 0,1 & - & 0,4 & 0,2 & - \\
\hline $6 \mathrm{Wav}$ & 94,9 & 1,2 & 0,2 & 2,2 & - & - & 0,1 & 0,2 & 0,1 & - & 0,1 & 0,1 & 0,1 & 0,1 & - \\
\hline $6 W n$ & 95,4 & 1,2 & 1,0 & 0,4 & 0,1 & - & - & 1,6 & - & - & - & 0,1 & - & 0,6 & - \\
\hline $6 \mathrm{Ws}$ & 88,2 & 1,4 & - & 6,6 & 0,2 & - & 0,6 & 0,2 & - & 0,2 & - & - & 0,2 & 0,2 & 0,1 \\
\hline $6 \mathrm{~B}$ & 65,9 & 5,6 & 10,6 & 1,4 & 8,0 & 0,1 & 1,6 & - & 0,2 & - & 0,6 & 0,2 & - & 0,2 & 4,0 \\
\hline 6D & 89,3 & 1,6 & 1,2 & 1,4 & 3,2 & - & 0,2 & - & 0,6 & - & 0,2 & 0,1 & - & 0,2 & 1,7 \\
\hline
\end{tabular}


Tableau 2. Suite.

Table 2. Continued.

\begin{tabular}{|c|c|c|c|c|c|c|c|c|c|c|c|c|c|c|c|}
\hline . & 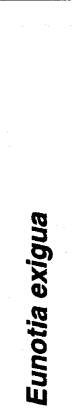 & 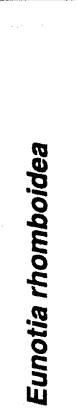 & 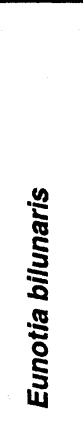 & 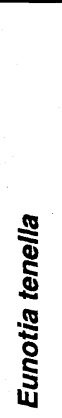 & 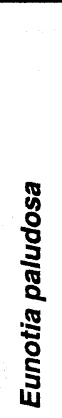 & 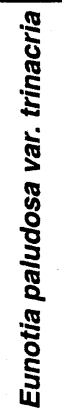 & 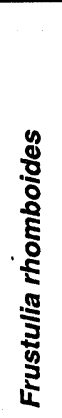 & 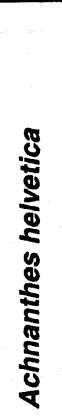 & 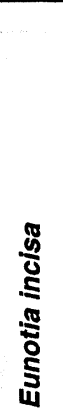 & 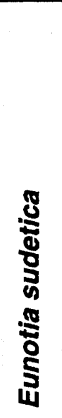 & 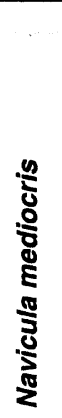 & 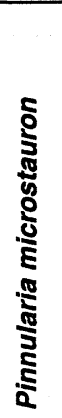 & 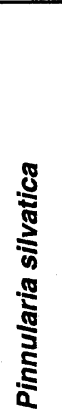 & 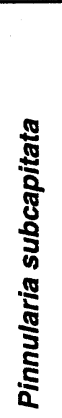 & 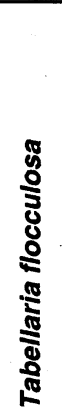 \\
\hline 7Rav & 70,6 & 14,4 & 7,8 & 0,2 & 0,4 & 0,6 & 2,3 & - & - & - & 0,4 & 0,2 & 0,6 & 0,8 & - \\
\hline 7Re1 & 76,2 & 5,8 & 5,0 & 0,4 & 5,8 & - & 0,1 & - & 0,4 & 0,1 & 0,4 & 0,6 & 0,2 & 3,2 & 0,4 \\
\hline 7Re2 & 74,6 & 2,4 & 5,6 & 1,2 & 5,6 & 0,4 & 0,1 & - & 0,1 & 0,2 & 0,2 & 0,6 & 0,2 & 5,6 & 0,1 \\
\hline 7Ro1 & 89,3 & - & 3,8 & 0,2 & 0,2 & 1,4 & 2,4 & 0,1 & - & - & 0,1 & - & 1,2 & 0,4 & - \\
\hline 7Ro2 & 82,6 & 0,6 & 6,6 & - & 1,0 & 2,0 & 1,3 & - & - & - & - & 1,0 & 0,4 & 3 & 0,1 \\
\hline 7Wav & 88,8 & 4,0 & 0,1 & 1,8 & 0,6 & 0,2 & 0,5 & 1,8 & 0,4 & - & 0,1 & 0,1 & 0,1 & 0,4 & 0,2 \\
\hline $7 W n$ & 91,9 & 2,8 & 0,1 & 0,8 & - & - & 0,2 & 1,6 & - & - & - & 0,2 & 0,2 & 1,6 & 0,1 \\
\hline $7 \mathrm{Ws}$ & 59,5 & 24,0 & 1,2 & 6,6 & - & - & 2,1 & 0,6 & - & 2,6 & - & - & 1 & 1,2 & - \\
\hline 7B & 73,2 & 19,0 & 1,0 & 0,2 & 4,5 & - & 0,2 & - & - & - & 0,2 & 0,2 & 0,1 & 0,2 & 0,6 \\
\hline 7D & 93,5 & 2,0 & 0,2 & 0,4 & 1,2 & - & 0,1 & - & - & - & 0,4 & 0,1 & - & 0,1 & 1,3 \\
\hline 8Rav & 49,2 & 46,2 & 2,8 & 0,1 & 0,1 & 0,2 & 0,2 & - & 0,1 & - & 0,4 & - & 0,1 & 0,2 & - \\
\hline $8 R e 1$ & 78,2 & 2,9 & 11,5 & 0,4 & 1,2 & - & 0,1 & - & 0,2 & 0,4 & 0,1 & 1,4 & - & 2,3 & 0,2 \\
\hline 8Re2 & 49,4 & 43,8 & 1,2 & 0,2 & 0,8 & - & - & 0,1 & 0,2 & 2,2 & 0,1 & - & 0,9 & 0,1 & 0,2 \\
\hline 8Ro1 & 94,1 & 0,4 & 2,8 & 0,2 & 0,1 & 0,1 & 1,4 & - & - & - & - & - & 0,2 & - & 0,1 \\
\hline 8Ro2 & 93,5 & 0,1 & 3,4 & 0,1 & 0,6 & 0,4 & 0,2 & - & - & - & - & 0,1 & 0,2 & 0,8 & 0,3 \\
\hline $8 \mathrm{Wav}$ & 82,8 & 13,6 & 0,6 & 0,2 & 0,4 & 0,1 & 0,2 & 0,1 & 0,1 & 0,1 & - & - & 0,1 & 0,4 & - \\
\hline $8 W n$ & 89,3 & 3,4 & 1,6 & 0,2 & 1,2 & 0,1 & 0,1 & - & 0,4 & - & 0,1 & - & - & 0,2 & 3,0 \\
\hline $8 W s$ & - & - & - & - & - & - & - & - & - & - & - & - & - & - & - \\
\hline $8 B$ & 47,4 & 28,2 & 3,6 & 0,8 & 3,8 & 0,2 & 0,9 & - & 0,1 & - & - & - & 0,1 & 0,1 & 13,8 \\
\hline 8D & 92,0 & 5,8 & 0,1 & - & 0,2 & - & 0,1 & 0,4 & 0,1 & - & 0,1 & 0,2 & - & 0,4 & 0,1 \\
\hline
\end{tabular}

\section{Travaux cités}

Alles E., Nörpel-Schempp M. \& Lange-Bertalot H. 1991. — Zur Systematik und Ökologie carakteristischer Eunotia-Arten (Bacillariophyceae) in elektrolytarmen Bachoberläufen. Nova Hedwigia, $53(1-2)$ : 171-213.

Anonyme 1994. - Restauration des forêts endommagées et suivi des effets. Projet pilote Région Wallonne - Union Européenne. Région Wallone : $56 \mathrm{p}$.

Battarbee R.W. 1984. - Diatom analysis and the acidification of lakes. Phil. Trans. r. Soc., Lond. B, 305 : 451-477.

Carnol M. 1997. - Etude comparée de paramètres écosystémiques et de l'évolution phytosanitaire de peuplements situés dans des couples de bassins versants. Convention entre la Région Wallonne et l'Université de Liège - Rapport final. Université de Liège, Laboratoire d'Ecologie Microbienne : $108 \mathrm{p}$.

Coring E. 1993. - Zum Indikationswert benthischer Diatomeengesellschaften in basenarmen Fliessgewässern. Aachen, Verlag Shaker : 165 p.

Fabri R. \& Leclercq L. 1984. - Etude écologique des rivières du nord du massif ardennais (Belgique) : flore et végétation des dia- tomées et physico-chimie des eaux. Stat. scient. Hautes-Fagnes, Robertville : vol. $1: 379$ p., vol. $2: 329$ p., vol. $3: 201$ p.

Kwandrans J. 1993. - Diatom communities of acidic mountain streams in Poland. Hydrobiologia, 269/270 (1-3) : 335-342.

Lancaster J., Real M., Juggins S., Monteith D.T., Flower R.J. \& Beaumont W.R.C. 1996. - Monitoring temporal changes in the biology of acid waters. Freshwater Biol., 36 : 179-201.

Pierre J.-F. 1996. - Communauté algale et acidité des ruisseaux du Massif Vosgien. Bull. Acad. Soc. lorr. Sci., 35(3) : 139-156.

Stevenson A.C., Juggins S., Birks H.J.B., Anderson D.S., Anderson N.J., Battarbee R.W., Berge F., Davis R.B., Flower R.J., Haworth E.Y., Jones V.J., Kingston J.C., Kreiser A.M., Line J.M., Munro M.A.R. \& Renberg I. 1991. - The Surface Waters Acidification Project Palaeolimnology Programme : Modern Diatom/Lake-water Chemistry Data-set. ENSIS, London : $86 \mathrm{p}$.

Symoens J.J. 1957. - Les eaux douces de l'Ardenne et des régions voisines : les milieux et leur végétation algale. Bull. Soc. roy. Bot. Belg., 89 : 111-314.

Van Dam H. 1981. - Impact of acidification on diatoms and chemistry of Dutch moorland pools. Hydrobiologia, 83 : 425-459. 